\title{
Some benefits of random variables in switched control systems
}

\author{
A. Allison*, D. Abbott \\ Department of Electrical and Electronic Engineering, University of Adelaide, Adelaide, SA 5005, Australia
}

\begin{abstract}
Recent advances in semiconductor technology have greatly increased the performance and range of application of switched mode circuits. Periodic switching can give rise to acoustic noise [Y.-S. Lai, Random switching techniques for inverter control, Electronics Letters 33 (9) (1977) 747-749] or undesirable electromagnetic radiation. These problems can be reduced through the use of random switching policies [S.Y.R. Hui, S. Sathiakumar, K.-K. Sung, Novel random pwm schemes with weighted switching decision, IEEE Transactions on Power Electronics 12(0885-8993) (1997) 945-951], but it is not always clear how this could be done without affecting other performance measures, such as RMS ripple or stability.

We use the buck/boost regulator as an example for analysis and determine some simple techniques for choosing appropriate component values. The circuit is simulated and it is shown that strict adherence to the formal limits of stability, suggested by control theory, does not always guarantee a satisfactory output.

We demonstrate that if switching is performed quickly enough then a state-space averaged model may be used for the buck/boost controller. This model is stable within wide bounds. It is possible to use some of this freedom to optimise EMC performance through the use of a control law which is random within certain limits.

In the popular mind, the idea of "randomness" seems to be completely opposed to the idea of "control." We show that not necessarily the case. Some randomness can beneficial, from the point of view of minimising the maximum power spectral density of the noise waveforms in the output current. This can be done without compromising the stability of the system. (C) 2000 Elsevier Science Ltd. All rights reserved.
\end{abstract}

Keywords: Switched mode; Control; Random; Stochastic resonance

\section{The buck/boost regulator as an example for analysis}

In this paper we use a state variable approach to systems, which is very general. Rather than discussing all systems purely in the abstract, we illustrate the important points using an example system. We have selected the buck/ boost regulator for the following reasons:

- It is very commonly used. Applications are found with the data sheets of many of the commercially available integrated circuits, such as the LM78S40.

- Simple analysis can readily be found in the literature $[1,2]$.

- Since this regulator can "boost" voltages, it has interesting stability properties. It can appear to be unstable if an inappropriate control rule is used.

- This regulator is composed from linear elements and can be readily formulated and analysed in state space.

The buck/boost regulator is basically a switched inductor circuit. The topology is in Fig. 1. The regulator has two

\footnotetext{
* Corresponding author.

E-mail address: aallison@eleceng.adelaide.edu.au (A. Allison).
}

modes. In mode 1 , called the "on" time, $\mathrm{S} 1$ is closed and $\mathrm{S} 2$ is open. In mode 2, called the "off" time, S1 is open and $\mathrm{S} 2$ is closed. We can denote the "on" time by $\Delta T_{1}$ and the "off" time by $\Delta T_{2}$. The use of the symbol " $\Delta$ " implies that the switching times are small compared with all of the time constants in the regulator.

This switched-mode system only has two modes and only a very simple control rule is needed, or even possible. The system can be viewed as a finite state machine and the control law can be represented using a state transition diagram, shown in Fig. 2. The "modes" of operation of the buck/boost regulator are shown as "states" of the state transition diagram.

In practice, $\mathrm{S} 1$ is often a bipolar transistor and $\mathrm{S} 2$ is a diode $[1,2]$. The buck/boost circuit is an inverting regulator. The average DC values of $V_{\mathrm{s}}$ and $V_{\mathrm{c}}$ are opposite in sign.

\section{Normal operating conditions and a simple approach to design}

The natural state variables to use for this type of circuit are the capacitor voltages and the inductor currents. 


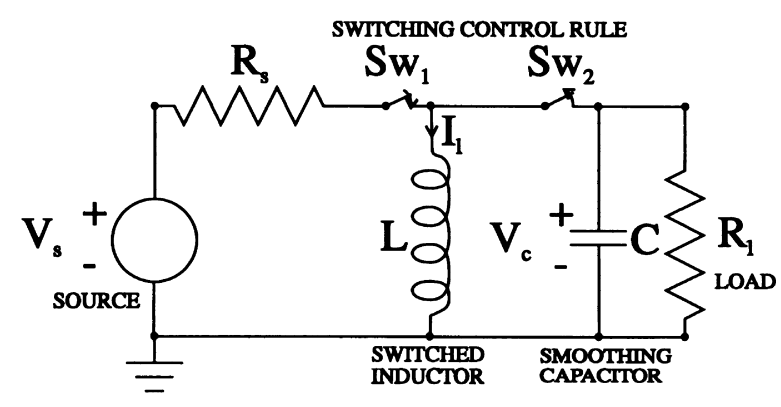

Fig. 1. A schematic circuit for the buck/boost regulator.

Together they characterise the total stored energy of the system. These variables are also preserved across switching boundaries.

If $\Delta T_{1}$ and $\Delta T_{2}$ are "small" then simulations show that the responses have a small triangular wave, or "ripple", superimposed on top of them. The rise and fall times of this superimposed wave are tied to the switching times, $\Delta T_{1}$ and $\Delta T_{2}$. If we consider the quiescent or DC case (after all transients have been attenuated), then we expect to get regular triangular waveforms, as shown in Fig. 3. We can develop a piecewise linear model.

The symbol $V_{\mathrm{c}}$ is used here to denote the median value of the capacitor voltage and $\Delta V_{\mathrm{c}}$ denotes the ripple voltage across the capacitor. The capacitor voltage is also equal to the output voltage, delivered to the load, $R_{1}$. Similarly, the symbol $I_{1}$ is used to denote the median value of the inductor current and $\Delta I_{1}$ denotes the ripple current through the inductor. During the "on" time, the inductor current is also equal to the input source current. During the "off" time, the input source current is zero.

The simple application of nodal and mesh equations to the system, in both modes, leads to the following formulation: during the "on" time:

$I_{1} R_{\mathrm{s}}+L \frac{\Delta I_{1}}{\Delta T_{1}}=V_{\mathrm{s}}$

$C \frac{\left(-\Delta V_{\mathrm{c}}\right)}{\Delta T_{1}}=\frac{V_{\mathrm{c}}}{R_{1}}$

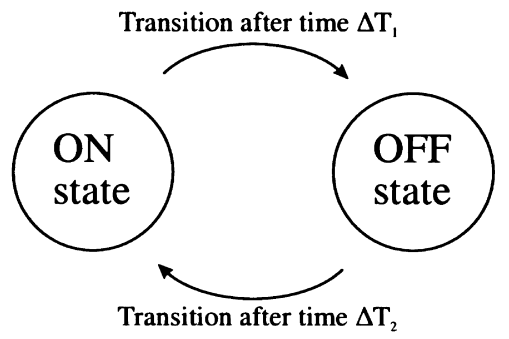

Switching time is $T_{s}=\Delta T_{1}+\Delta T_{2}$ (can vary within limits)

Duty cycle $=\Delta \mathrm{T}_{1} /\left(\Delta \mathrm{T}_{1}+\Delta \mathrm{T}_{2}\right)$ (use this to control output voltage)

Fig. 2. A simple control rule for the buck/boost controller.

\section{Simple Piecewise Linear Model}
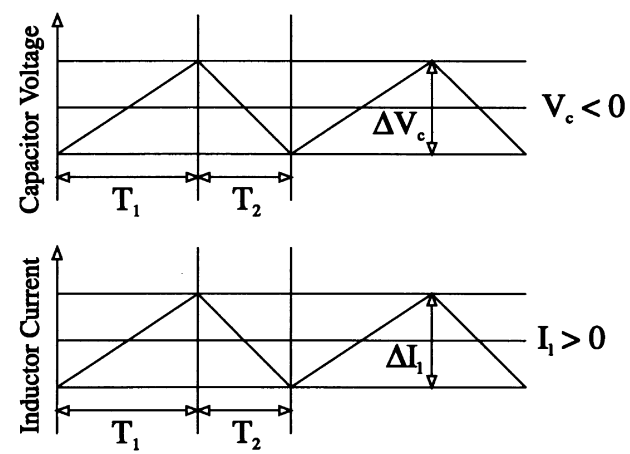

Fig. 3. A piecewise linear model for the buck/boost regulator.

and during the "off" time:

$I_{1}-C \frac{\Delta V_{\mathrm{c}}}{\Delta T_{2}}+\frac{V_{\mathrm{c}}}{R_{1}}=0$

$-L \frac{\Delta I_{1}}{\Delta T_{2}}=V_{\mathrm{c}}$

We can eliminate terms involving $\Delta V_{\mathrm{c}}$ and $\Delta I_{1}$ and write the equations in matrix form:

$$
\begin{aligned}
& {\left[\begin{array}{cc}
\left(\frac{-1}{R_{1} C}\right) & \left(\frac{-(1-d)}{C}\right) \\
\left(\frac{+(1-d)}{L}\right) & \left(\frac{-d R_{\mathrm{s}}}{L}\right)
\end{array}\right]\left[\begin{array}{c}
V_{\mathrm{c}} \\
I_{1}
\end{array}\right]+\left[\begin{array}{c}
0 \\
\left(\frac{V_{\mathrm{s}} d}{L}\right)
\end{array}\right]} \\
& =\left[\begin{array}{l}
0 \\
0
\end{array}\right]
\end{aligned}
$$

where $d$ is the time averaged duty cycle, $\left(\Delta T_{1} /\left(\Delta T_{1}+\right.\right.$ $\left.\Delta T_{2}\right)$ ).

We can solve the equations algebraically and derive an expression for $V_{\mathrm{c}}$ in terms of the source voltage, $V_{\mathrm{s}}$, the ratio of the switching times, $\left(\Delta T_{1} / \Delta T_{2}\right)$ and the ratio of the source and load impedances, $\left(R_{\mathrm{S}} / R_{l}\right)$ :

$V_{\mathrm{c}}=\frac{\left(-V_{\mathrm{s}}\right)}{1 /\left(\Delta T_{1} / \Delta T_{2}\right)+\left(\frac{R_{\mathrm{s}}}{R_{1}}\right)\left(\left(\frac{\Delta T_{1}}{\Delta T_{2}}\right)+1\right)}$.

It is usually the case that $\left(R_{\mathrm{S}} / R_{1}\right)$ is very small so we can write

$V_{\mathrm{c}} \approx\left(-V_{\mathrm{s}}\right)\left(\frac{\Delta T_{1}}{\Delta T_{2}}\right)$

and can readily control the median output voltage by controlling the duty cycle of the switching control rule.

After the component values have been fixed, we can still control the behaviour of the output by making changes to the control rule, shown in Eq. (2). We can vary the amount of ripple by controlling $T_{\mathrm{s}}=\Delta T_{1}+\Delta T_{2}$ and also vary the median output voltage by controlling $\left(\Delta T_{1} / \Delta T_{2}\right)$, as suggested by Eq. (7). 
If the source impedance $R_{\mathrm{s}}$ is appreciable, then $V_{\mathrm{c}}$ has an upper bound. This occurs when

$\left(\frac{\Delta T_{1}}{\Delta T_{2}}\right)=\frac{1}{\sqrt{R_{\mathrm{s}} / R_{1}}}$

and we get

$\max \left|V_{\mathrm{c}}\right|=\frac{\left(-V_{\mathrm{s}}\right)}{2 \sqrt{\left(R_{\mathrm{s}}\right) /\left(R_{1}\right)}+\left(R_{\mathrm{s}} / R_{1}\right)}$.

If $\left(R_{\mathrm{S}} / R_{1}\right)$ is small, then we can write

$\max \left|V_{\mathrm{c}}\right| \approx \frac{1}{2} \frac{V_{\mathrm{s}}}{\sqrt{\left(R_{\mathrm{s}} / R_{1}\right)}}$.

We can still control $V_{\mathrm{c}}$ within this range by altering the duty cycle.

We can readily calculate the amount of ripple, $\Delta V_{\mathrm{c}}$

$\Delta V_{\mathrm{c}}=\left(-V_{\mathrm{c}}\right) \frac{\Delta T_{1}}{R_{1} C}$

The designer must make some decision about the amount of ripple that can be tolerated. Eqs. (6) and (8) can be used to estimate appropriate values for $C$ and the initial design value of $\left(\Delta T_{1} / \Delta T_{2}\right)$.

We can also solve for the current, $I_{1}$ and the ripple current $\Delta I_{1}$

$I_{1}=\frac{V_{\mathrm{s}}}{R_{1} /\left(\Delta T_{1} / \Delta T_{2}\right)\left(\left(\Delta T_{1} / \Delta T_{2}\right)+1\right)+R_{\mathrm{s}}}$.

$\Delta I_{1}=\left(-V_{\mathrm{c}}\right) \frac{\Delta T_{2}}{L}=\frac{\left(-V_{\mathrm{c}}\right)}{R_{1}} \frac{\Delta T_{2}}{\left(L / R_{1}\right)}=I_{\mathrm{out}} \frac{\Delta T_{2}}{\left(L / R_{1}\right)}$

Eqs. (9) and (10) can be used to select the inductance and the current rating of the inductor.

It was assumed that the regulator would be supplied by a source with the nominal parameters of $R_{\mathrm{s}}=0 \Omega$ and $V_{\mathrm{s}}=$ $+10 \mathrm{~V}$. A modest and very feasible switching time of $25 \mu \mathrm{s}$ was chosen. Using the simple approach, a system was designed to guarantee that $V_{\mathrm{c}}=-10.0 \mathrm{~V}$ and $\Delta V_{\mathrm{c}}=$ $10 \mathrm{mV}$. The percentage ripple in $I_{1}$ was designed to be about the same at the percentage ripple in $V_{\mathrm{c}}$. The selected values were: $C=1500 \mu \mathrm{F}$ and $L=68 \mathrm{mH}$. It is reassuring that these component values are not extreme. The actual numerical values are less important than the procedure used in the design, which is quite general.

The simple, piecewise linear, model does allow some estimates of currents in the inductor and in the load and their rates of change. The main sources of EMI, in switch mode supplies, are the square switching waveforms, the charging current at the input and the current at the load $[3,4]$. If we are to estimate these quantities more accurately, then we need a more realistic dynamical model. We also need to have a more accurate model to study the transient behaviour and the stability of these circuits. The most widely accepted and general approach to this problem is to use state variable techniques [5-7].

\section{A state variable formulation of a switched mode circuit}

If we apply infinitesimal differentials to the analysis of the buck/boost regulator, rather than finite differences, then Eqs. (1)-(4) can be rewritten using matrix notation.

For the "on" mode, we get:

$\left[\begin{array}{c}\dot{V}_{\mathrm{c}} \\ \dot{I}_{1}\end{array}\right]=\left[\begin{array}{cc}\left(-1 / R_{1} C\right) & 0 \\ 0 & \left(-R_{\mathrm{s}} / L\right)\end{array}\right]\left[\begin{array}{c}V_{\mathrm{c}} \\ I_{1}\end{array}\right]+\left[\begin{array}{c}0 \\ \left(V_{\mathrm{s}} / L\right)\end{array}\right]$

For the "off" mode, we get:

$\left[\begin{array}{c}\dot{V}_{\mathrm{c}} \\ \dot{I}_{1}\end{array}\right]=\left[\begin{array}{cc}\left(-1 / R_{1} C\right) & (-1 / C) \\ (1 / L) & 0\end{array}\right]\left[\begin{array}{c}V_{\mathrm{c}} \\ I_{1}\end{array}\right]+\left[\begin{array}{l}0 \\ 0\end{array}\right]$.

For the "on" mode, we get: $\dot{X}=A_{1} X+U$. For the "off" mode, we get: $\dot{X}=A_{2} X+0$. The column vector $X$ contains the state variables. There are two different transition matrices, $A_{1}$ and $A_{2}$. The column vector, $U$ represents the input from the source.

The state variables have been deliberately chosen in such a way that they are preserved across the switching boundaries. At these switching moments, the state transition matrices $A_{1}$ and $A_{2}$ are substituted for a single matrix.

\section{Stability of the circuit in each mode}

The system is linear in either mode and the stability can be readily analysed by examining the eigenvalues of the transition matrices. If we invoke the theory of the Laplace transform then these eigenvalues can be identified with the poles of the system in the $s$ domain. The eigenvalues of $A_{1}$ are: $\left(-1 / R_{1} C\right)$ and $\left(-R_{\mathrm{s}} / L\right)$. The eigenvalues of $A_{2}$ are: $-\sigma+j \omega_{\mathrm{d}}$ and $-\sigma-j \omega_{\mathrm{d}}$, where

$\zeta=\frac{1}{2} \frac{+\sqrt{L / C}}{R_{1}}, \quad \omega_{0}=\frac{1}{+\sqrt{L C}}$

$\sigma=+\omega_{0} \zeta$ and $\omega_{\mathrm{d}}=\omega_{0}\left(+\sqrt{1-\zeta^{2}}\right)$. The variable, $\zeta$ is called the damping factor and $\omega_{\mathrm{d}}$ the damped frequency of oscillation, in radians per second. All of these eigenvalues have negative real parts and so the system is stable in either mode.

\section{Some simulation results}

The state-space equations can be solved exactly using the theory of the Laplace transform [8]. The general solution is of the form:

$x(t)=\exp (A t) x(0)+\int_{0}^{t} \exp [A(t-\tau)] u(t) \mathrm{d} t$.

The $\exp ($ ) function refers to the matrix power series

$\exp (A t)=\sum_{k=0}^{\infty} \frac{(A t)^{k}}{k !}$ 

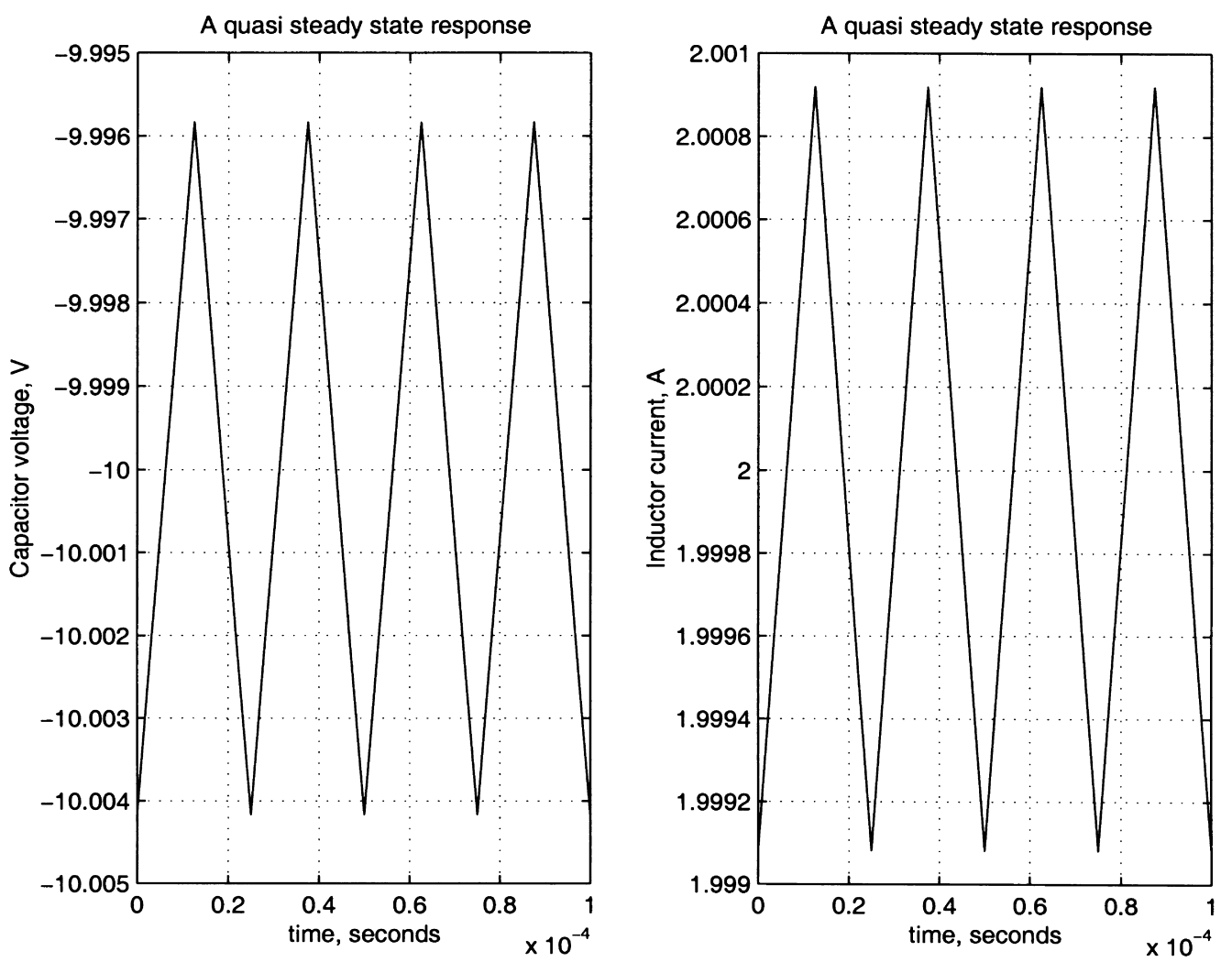

Fig. 4. State-space simulation of the almost quiescent case.
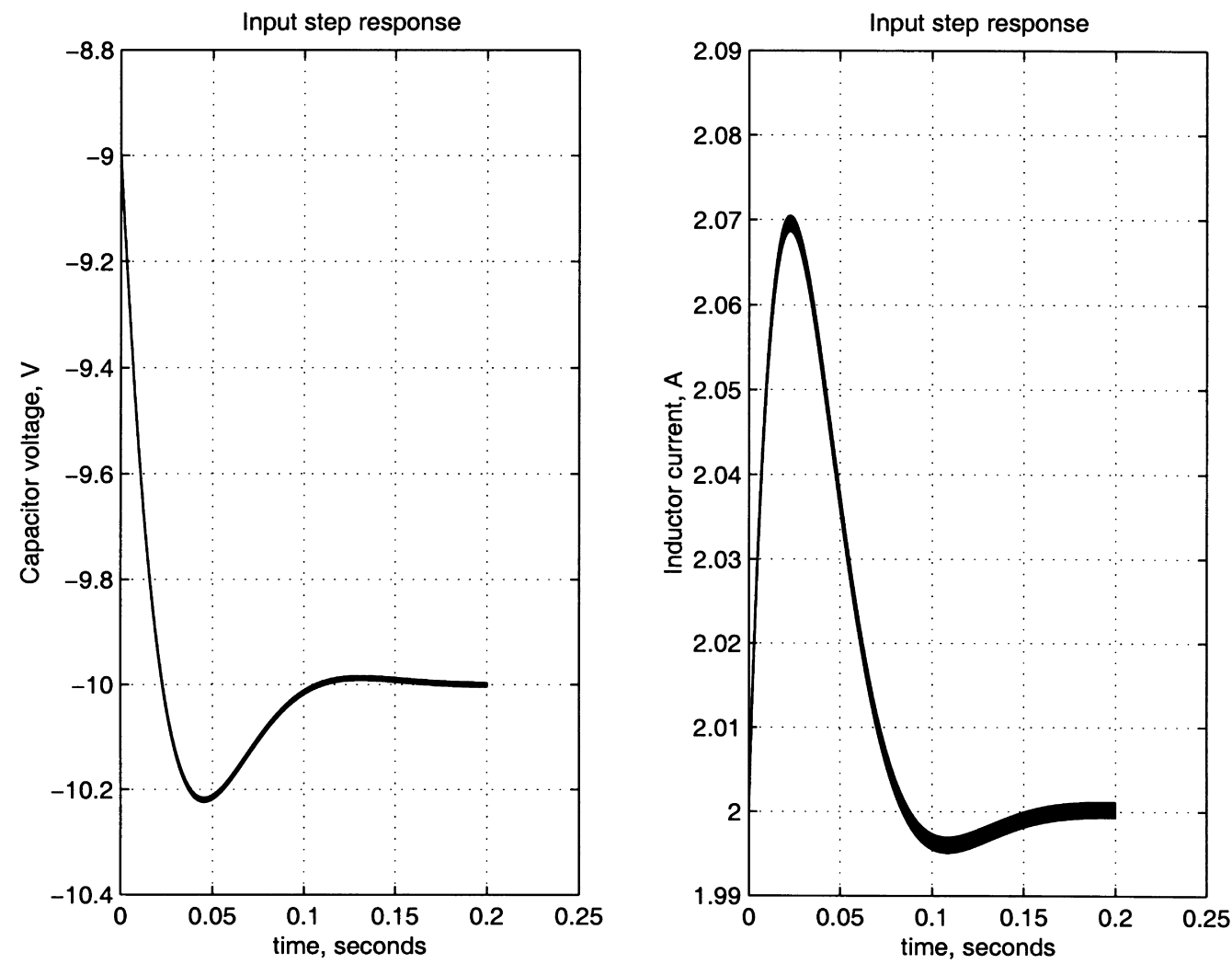

Fig. 5. State-space simulation of a transient response to a step change in the load. 

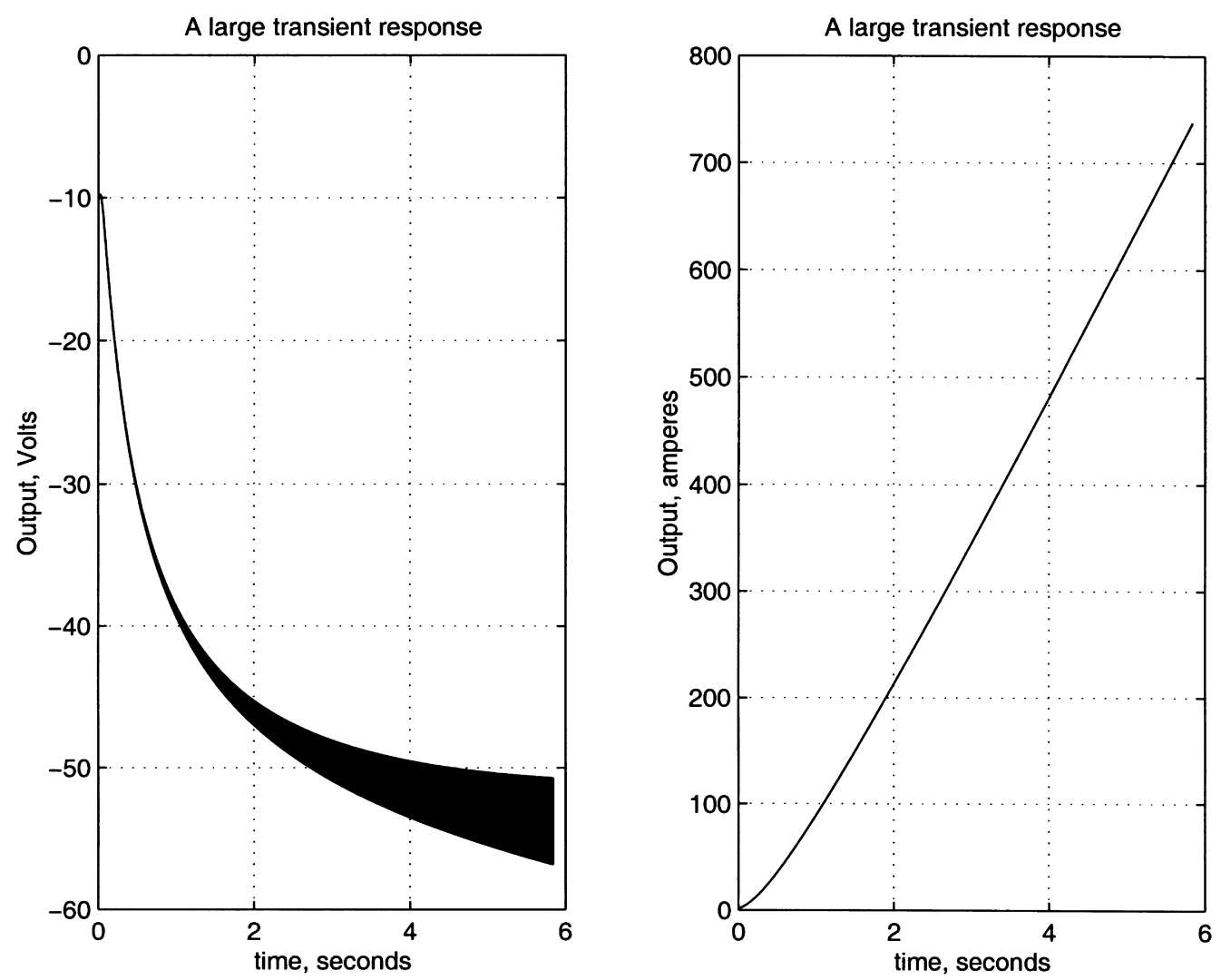

Fig. 6. State-space simulation of very large, almost unstable, transient response.

and the second term follows from the convolution property of the Laplace transform. If we perform the integration directly and apply Eq. (13) to two adjacent time intervals $\Delta T_{1}$ and $\Delta T_{2}$, shown in Fig. 2, then we get an equation that describes the evolution of the system in the time domain:

$$
\begin{aligned}
X\left(t+\Delta T_{1}+\Delta T_{2}\right)= & \left(\exp \left(A_{2} \Delta T_{2}\right) \exp \left(A_{1} \Delta T_{1}\right)\right) X(t) \\
& +\left(\Delta T_{1}\right) \exp \left(A_{2} \Delta T_{2}\right)\left(A_{1} \Delta T_{1}\right)^{-1} \\
& \times\left(\exp \left(A_{1} \Delta T_{1}\right)-I\right) U
\end{aligned}
$$

It is possible to simulate Eq. (14) directly and quite accurately on a digital computer using mathematical software, such as Matlab. The almost quiescent case was described by the piecewise linear model. It is important to check that the state-space model is consistent with this model. The simulation is shown in Fig. 4. This is consistent with what we expect from the simple model.

We wish to examine the way in which the system might respond to a sudden change in load, in the absence of any other changes. This was simulated and is shown in Fig. 5.

Clearly this response is stable. This gives us some confidence that even very simple control laws, such as fixed duty cycle, may be useful. We would hope that the duty cycle and frequency could be altered by the control law at a rate which is slow in comparison with the switching operation.

Eqs. (6) and (7) suggest that we could make $V_{\mathrm{c}}$ arbitrarily large by changing the duty cycle. This suggests the following very simple control law: At each switching moment $\Delta T_{2}$ is preserved and $\Delta T_{1}$ is increased: $\Delta T_{1} \leftarrow(1+\epsilon) \Delta T_{1}$. We chose $\epsilon=0.0003$. The result is shown in Fig. 6. The apparent thickness of the lines in Fig. 6 is not an artefact of the numerical method. It is due to "zig-zag" ripple waveforms, similar to those shown in Fig. 4. It is a fundamental part of the dynamical behaviour of the circuit.

The response shown in Fig. 6 appears to be unstable. A more detailed analysis shows that the eigenvalues of $\left(\exp \left(A_{2} \Delta T_{2}\right) \exp \left(A_{1} \Delta T_{1}\right)\right)$ have absolute values of less than 1 so that this response is not "unstable" in the technical sense. The response should be bounded.

There are two fairly clear lessons to be drawn from this peculiar transient response.

1. Strict reliance on proofs of stability is often found in the literature $[8,9]$. These theorems are very formal and are ultimately true but they may not always be useful in practice. The response may become completely unsatisfactory long before it becomes "unstable" in the technical sense.

2. We need a very simple criterion to help us to stay out of danger. Eqs. (8) and (10) suggest that the switching times should be small compared with the time constants, $\left(R_{1} C\right)$ and $\left(L / R_{1}\right)$. The definition of "small" should come from a specification of quality of the state variables. The 

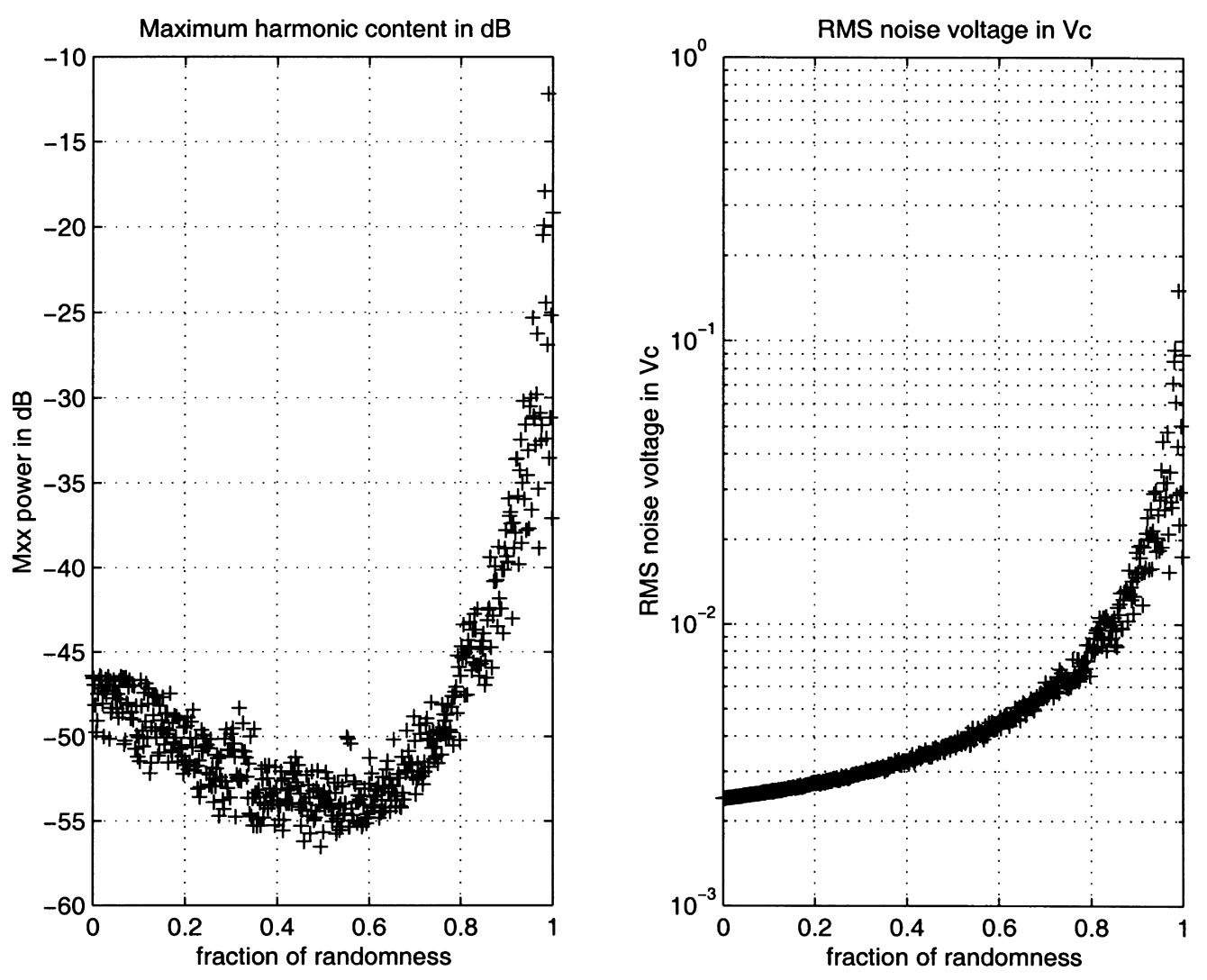

Fig. 7. Variation of quality performance measures in response to a random factor, $\rho$.

requirement that the output ripple voltage be small is a much more stringent condition than the requirement that the output be stable.

\section{The state-space averaged model}

The simulations in this paper were all achieved using a direct evaluation of the switched state-space model. If we imagine a Boolean switching function, $q(t) \in\{0,1\}$, then we could write a single dynamical equation:

$\dot{X}=\left(A_{1} q(t)+A_{2}(1-q(t))\right) X+B V_{\mathrm{s}}$

From a pure mathematical point of view, the switching function, $q(t)$, could be arbitrarily complicated. It could have infinite bandwidth. In practice, the fine structure of $q(t)$ does not matter very much since it is averaged by the plant. We can imagine a time averaged switching function:

$d(t)=\frac{1}{T_{\mathrm{s}}} \int_{t-\tau}^{t} q(\tau) \mathrm{d} \tau$.

In general, $d(t)$ would be a real function on the interval $[0,1]$. We could then write:

$\dot{X}=\left(A_{1} d(t)+A_{2}(1-d(t))\right) X+B V_{\mathrm{s}}=A_{3} X+B V_{\mathrm{s}}$

This is called the state-space averaged model [10]. It is a valid approximation as long as we choose an appropriate time frame, $T_{s}$. Strictly speaking, the averaging should occur in the time domain. We should average the matrix $\left(\exp \left(A_{2} \Delta\right.\right.$ $\left.\left.T_{2}\right) \exp \left(A_{1} \Delta T_{1}\right)\right)$ and compare this with an equivalent matrix $\exp \left(A_{3}\left(\Delta T_{1}+\Delta T_{2}\right)\right)$. The result is the same as averaging in state space as long as $\Delta T_{2}$ and $\Delta T_{1}$ are small. We could choose $T_{\mathrm{s}}$ to represent the last switching cycle, in which case we get $d(t)=\Delta T_{1} /\left(\Delta T_{1}+\Delta T_{2}\right)$, which is the duty cycle. We need to choose $T_{\mathrm{s}}$ to be small compared with the time constants of the plant but this still leaves us with considerable free choice for $T_{\mathrm{s}}$ and $d(t)$.

The stability of the switched system can be established by examining the eigenvalues of the time averaged transition matrix, $A_{3}=\left(A_{1} d(t)+A_{2}(1-d(t))\right)$. For the buck/boost regulator, the real parts of the eigenvalues are:

$-\frac{1}{2}\left(\frac{1}{R_{1} C}+\frac{R_{\mathrm{s}}}{L} d(t)\right)$

which are always negative so the switched system is always stable as long as we switch quickly enough for the time averaged state-space model to be valid.

\section{Some simulations using random variables in the control rule}

For the buck/boost regulator, we have a very simple stability result. The system is stable as long as the switching 

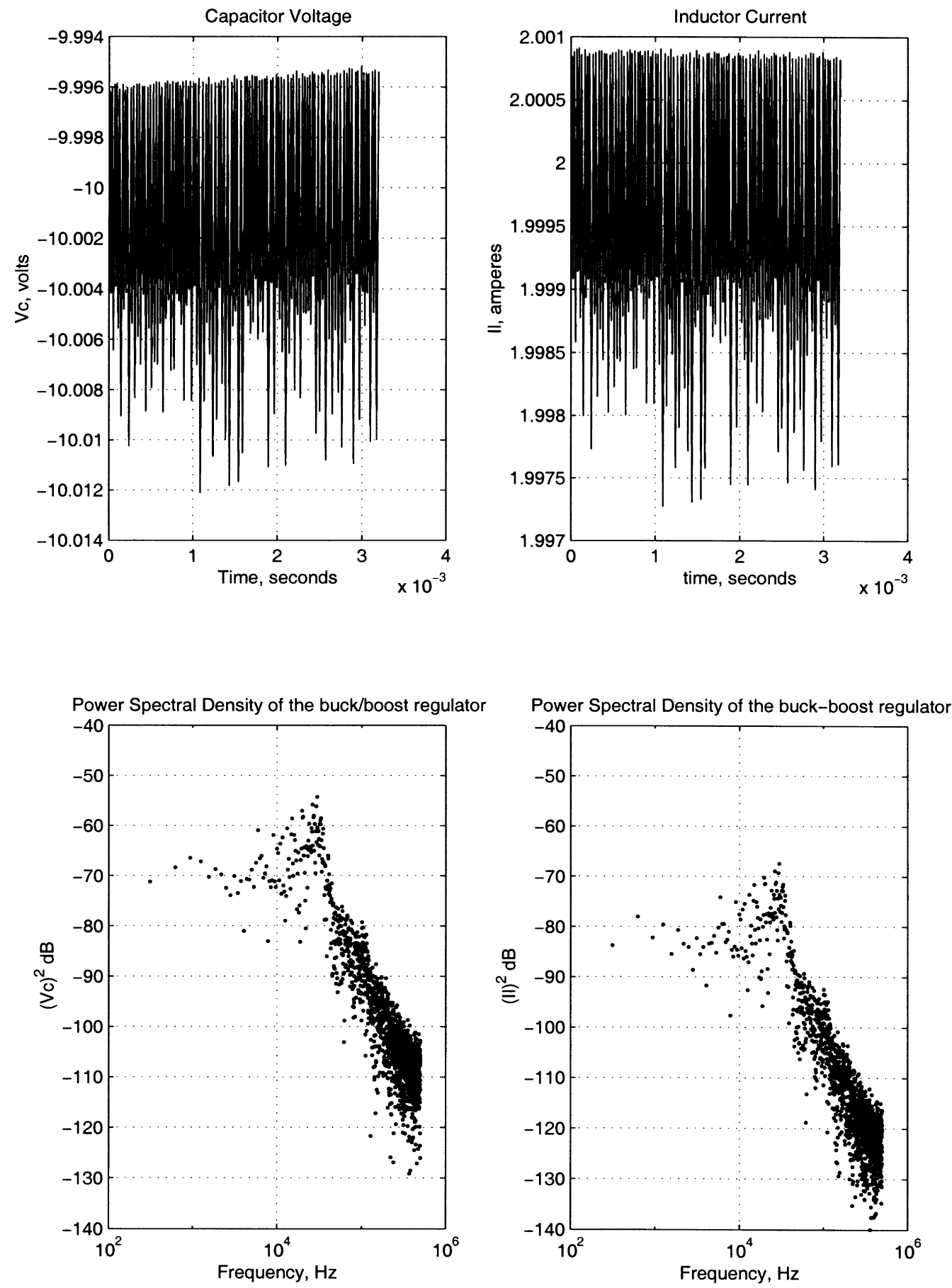

Fig. 8. Transient behaviour and spectral density of randomly switched response.

frequency is high enough. We would like to use this freedom to improve the performance of the regulator.

We know that periodic switching can give rise to undesirable electromagnetic radiation and that the EMC standards refer to maximum power spectral density [4]. Hui [11] has shown that the use of random switching functions can improve the spectral characteristics of power inverters. We propose an extremely simple random switching rule for the variation of the switching frequency. We choose $T_{\mathrm{s}}=(\rho \xi+(1-\rho)) * T_{0}$. The parameter $\rho$ is a real number in the range $0<\rho<1$. It represents a degree of randomness or random factor but is not a random variable. The parameter $T_{0}$ is the maximum value that the switching time, $T_{\mathrm{s}}$, may take. The variable $\xi$ is a uniform random variable in the range $0<\xi<1$. This scheme guarantees that the switching frequency always lies in the interval $\left(1 / T_{0}\right)<F_{\mathrm{s}}<\left(1 /\left((1-\rho) * T_{0}\right)\right)$. We can choose $T_{0}$ in order to satisfy the quality requirement for ripple voltage. 
This will also guarantee stability. We study the way in which RMS noise ripple voltage and maximum power spectral density, vary in response to changes in $\rho$. The results are shown in Fig. 7.

The EMC performance shows a qualitative similarity to stochastic resonance [12]. The EMC performance improves as we increase the random factor, $\rho$, up to a value of about 0.5 . It is possible to get a $5-10 \mathrm{~dB}$ improvement in performance. This is paid for by a small but steady increase in the RMS ripple voltage. Eventually, the output becomes overwhelmed by noise and the EMC performance degrades.

The performance of this control law with $\rho=0.5$ is shown in Fig. 8. We should note that this is still basically the same control law, shown in Fig. 2. The only difference is that we allow $T_{\mathrm{s}}=\Delta T_{1}+\Delta T_{2}$ to vary within limits while controlling the value of $d=\Delta T_{1} / \Delta T_{2}$.

\section{Summary and conclusions}

The simple piecewise linear approach to design was found to be adequate and agreed quite well with the results from the more complex state-space model. The switching times must be small in relation to the time constants of the system. If this condition is ever violated then the transient responses can be very large, even though the system is stable. The use of fast switching times allows the use of the time averaged state-space model.

If we use fast switching times then we have some free choice of the switching frequency. We can use this freedom to improve EMC performance of the system by using some randomness in the control law. This can be done without compromising stability. The use of "too much" randomness eventually degrades the output of the regulator. Provided that it is used within limits, some randomness can be beneficial.

\section{References}

[1] K. Billings, Switchmode Power Supply Handbook, McGraw-Hill, New York, 1989.

[2] J.C. Whitaker, The Electronics Handbook, CRC Press, West Palm Beach, FL, 1996.

[3] M.K.W. Wu, C.K. Tse, A review of emi problems in switch mode power supply design, Journal of Electrical and Electronics Engineering Australia 16 (1996) 193-204.

[4] T. Williams, EMC for product designers, Newnes, 1996.

[5] P.M. Derusso, R.J. Roy, C.M. Close, State Variables for Engineers, Wiley, New York, 1965

[6] L.O. Chua, P.M. Lin, Computer Aided Analysis of Electronic Circuits, Prentice-Hall, Englewood Cliffs, NJ, 1975.

[7] R.C. Dorf, R.H. Bishop, Modern Control Systems, Addison-Wesley, Reading, MA, 1998.

[8] D. Něsć, E. Skafidas, I.M.Y. Mareels, R.J. Evans, Minimum phase properties for input nonaffine nonlinear systems, IEEE Transactions on Automatic Control 44 (4) (1999) 868-870.

[9] E. Skafidas, R.J. Evans, A.V. Savkin, I.R. Petersen, Stability results for switched controller systems, Automatica 35 (4) (1999) 553-564.

[10] G.C. Verghese, D.G. Taylor, T.M. Jahns, R.W.D. Doncker, Power Electronic Controls, CRC Press and IEEE Press, 1996.

[11] S.Y.R. Hui, S. Sathiakumar, K.-K. Sung, Novel random pwm schemes with weighted switching decision, IEEE Transactions on Power Electronics 12(0885-8993) (1997) 945-951.

[12] F. Moss, K. Wiesenfield, The benefits of background noise, Scientific American 273 (2) (1995) 50-53. 